\title{
Clasificación de perfiles de uso de smartphones en estudiantes y docentes de la Universidad Autónoma de Baja California, México
}

\section{Classification of use profiles of smartphones in students and teachers of Autonomous University of Baja California, México}

\author{
Patricio HENRÍQUEZ RITCHIE ${ }^{1}$, Coral GONZÁLEZ BARBERA² ${ }^{2}$ \\ Javier ORGANISTA SANDOVAL ${ }^{1}$ \\ Universidad Autónoma de Baja California ${ }^{1}$ y Universidad Complutense de Madrid $^{2}$
}

Recibido: Febrero 2013

Aceptado: Mayo 2013

\section{Resumen}

El uso de recursos tecnológicos portátiles en general, y de teléfonos inteligentes (smartphones) en particular, es un fenómeno social en aumento durante la última década, especialmente entre las nuevas generaciones. A partir de la multiplicidad de medios y aplicaciones que ofrecen, principalmente referidas a la comunicación, manejo de información y de medios, se vislumbra un gran potencial educativo, lo que ha originado el desarrollo del concepto aprendizaje móvil. El presente artículo propone una clasificación de perfiles de uso de smartphones a partir de una muestra representativa de estudiantes y docentes de la Universidad Autónoma de Baja California (UABC), México. Para esto, se llevó a cabo un análisis clasificatorio de conglomerados (K-Means Clusters). Los resultados indician que los perfiles identificados con un nivel de uso avanzado se relacionan con un mayor porcentaje de uso educativo asignado al smartphone, un mejor autoconcepto ante el uso de la tecnología en general, un mejor dominio del idioma inglés y mayores niveles de pericia (frecuencia, percepción y habilidad) de uso de tales recursos.

Palabras clave: Aprendizaje móvil; clasificación de uso de teléfonos inteligentes; m-learning; uso educativo de teléfonos inteligentes.

\begin{abstract}
The use of portable technological resources in general and smartphones in particular, is a growing social phenomenon in the last decade, especially among the younger generations. From the diversity of media and applications it offers, and especially with its capabilities in communication and management of information and media, smartphones are seen as a great educational potential. Such capabilities led to the development of the mobile learning concept. This paper proposes a classification of use profiles of smartphones from a representative sample
\end{abstract}


of students and teachers of the Autonomous University of Baja California (UABC), Mexico. It performed a cluster analysis (K-Means Clusters). The results indicate that advanced use profiles relate to a higher percentage of educational use assigned to the smartphones, higher self-concept to the use of technology in general, a better management of English language and higher levels of expertise (frequency, perception and ability) of use of these resources.

Keywords: Mobile learning; educational use of smartphones; classification of use of smartphones.

La relación entre el ámbito educativo y el desarrollo tecnológico ha estado acechada por intensos debates y discusiones acerca de su sentido, trascendencia e impacto de uno sobre otro. Como destaca Cabero (2003), por un lado está el determinismo tecnológico, que defiende la idea de que la tecnología determina el desarrollo de la sociedad, marcando el ritmo de la historia humana y su evolución. Por otro lado, aparece la corriente Ciencia, Tecnología y Sociedad (CTS) que muestra una visión holística entre la tecnología y todos los ámbitos de la sociedad, entre ellos el educativo. Desde esta perspectiva, el desarrollo tecnológico no determina ni es determinado por el ámbito educativo. Ambas partes confluyen, interaccionan y se influyen recíprocamente, sin que exista una supremacía de una sobre la otra.

Durante la primera década del siglo XXI se ha observado un incremento superlativo en la posesión y uso de recursos tecnológicos portátiles, tales como asistentes personales digitales (PDA), dispositivos de almacenamiento de datos (USB, MP3, MP4), teléfonos celulares, tabletas, teléfonos inteligentes (smartphones), entre otros. Los datos al respecto son contundentes: la posesión de teléfonos celulares alcanzó a un cuarto de la población mundial (1,520 millones de usuarios) y en el caso específico de España, superó el $107 \%$ de la población total, existiendo más celulares que habitantes (Gómez y Martínez, 2008; Brazuelo y Cacheiro, 2010). Paulatinamente, la telefonía móvil se está convirtiendo en una base fundamental para la difusión de información y comunicación en la sociedad, principalmente en el denominado primer mundo.

De los recursos tecnológicos portátiles existentes, el presente artículo se interesa en uno particularmente: el teléfono inteligente o smartphone. Dado que en este recurso converge la telefonía móvil con la tecnología computacional, el abanico de posibilidades para utilizarlo como herramienta de apoyo a los procesos de enseñanzaaprendizaje es amplio. En efecto, la posibilidad de acceder a la red de manera inalámbrica (vía 3G/4G, Wi-Fi) para buscar, consultar, manejar y compartir información, sus múltiples vías de comunicación (llamadas por voz, mensajería instantánea, correo electrónico, foros asincrónicos, videoconferencias, chats, redes sociales), el manejo de medios (captura/reproducción/edición de audio, fotos y videos), aplicaciones informáticas (procesador de texto, hojas de cálculo), además de aplicaciones de organización (agenda digital, recordatorios, editor de notas, calculadora, calendario, etc.), manifiestan su gran potencial educativo, lo cual puede beneficiar las actividades académicas de estudiantes y docentes.

Entre la comunidad científica internacional ha surgido el interés por explorar el potencial pedagógico de la tecnología portátil, erigiéndose el denominado aprendizaje móvil (mobile learning, m-learning). Debido a su desarrollo incipiente, aún no se han 
logrado consolidar los pilares teóricos y metodológicos para su implementación en los procesos de enseñanza-aprendizaje. Sobre la base de los antecedentes anteriores, este artículo analiza la posesión y uso de smartphones en una muestra representativa de estudiantes y docentes de una universidad pública de México (Universidad Autónoma de Baja California, UABC).

\section{Objetivo}

Establecer una clasificación de perfiles de uso de smartphones en estudiantes y docentes de una universidad pública mexicana (UABC), a partir de variables personales, socioeconómicas y académicas, que sirva de base para su incorporación como herramienta de apoyo a las actividades académicas de ambos.

\section{Referentes teóricos}

El aprendizaje móvil surge del interés por explorar de qué manera los recursos tecnológicos portátiles pueden complementar y apoyar los procesos educativos. Sin embargo, son muchos los desafíos a los que se enfrenta. Uno de los principales desafíos es de carácter tecnológico. La multiplicidad de recursos portátiles existentes, sumada a la rapidez con que estos recursos se van renovando y transitando (dejando obsoletos en poco tiempo los equipos que aparecen en el mercado), dificulta el desarrollo teórico y metodológico para su implementación en el ámbito educativo (Kukulska-Hulme, 2009; Traxler, 2009). De esta manera, es evidente que centrarse en un punto de vista exclusivamente tecnológico (tecnocentrismo) no es el camino más adecuado para definir al aprendizaje móvil.

Otros desafíos importantes a los que se enfrenta el aprendizaje móvil son de corte pedagógico. Al respecto, Sharples (2005) advierte acerca de las estructuras y métodos en los que se basa la enseñanza tradicional: dimensiones espaciales y temporales fijas (salón de clases, horas de clase), recursos didácticos comunes, el docente como epicentro del proceso educativo, el estudiante en su rol de receptor pasivo de la información que le proporciona el docente, junto con un férreo control de los recursos tecnológicos que pueden utilizarse dentro de los límites espacio-temporales dispuestos. Todas estas características distan mucho del contexto en el que se pueden desarrollar las actividades educativas apoyadas con recursos tecnológicos portátiles, entre ellos los smartphones.

Sobre la base de lo anterior, Sharples (2007) vislumbra una confrontación entre dos sistemas de actividad en el ámbito educativo. Por un lado, el sistema de educación escolar tradicional, regulado por un currículum fijo y evaluaciones tradicionales. Este sistema se basa en clases presenciales protagonizadas por el docente, quien establece las formas de discurso aceptables y donde el acceso sin regulación a la información y comunicación posibilitado por Internet representa una amenaza al orden escolar. Por otro lado, los sistemas de redes sociales, mediados por perfiles personales y programas de interacción social entre individuos. Las nuevas generaciones van creando una nueva realidad basada en el intercambio de información, libertad de expresión, creatividad de 
lenguaje e interacción social, que se escapa del control ejercido en el sistema educativo tradicional.

A su vez, los desafíos pedagógicos del aprendizaje móvil se relacionan con las nuevas posibilidades de uso que propicia la tecnología portátil. Éstas se definen por su portabilidad, lo que remite a sus dimensiones físicas y la consecuente posibilidad de utilizarlos de manera espontánea e inmediata. Y por la movilidad asociada a los usuarios, lo que permite su uso en cualquier momento, lugar y contexto (Naismith, Lonsdale, Vavoula y Sharples, 2005). En efecto, a partir de estos elementos se vislumbran los desafíos para el desarrollo del proceso de enseñanza-aprendizaje móvil, pues justamente la portabilidad de los recursos tecnológicos y la posibilidad de utilizarlos en un contexto de movilidad constante generan ciertas tensiones con las dinámicas educativas tradicionales.

En torno a su conceptualización, Koole (2006; 2009) propone una estructura teórica para entender el aprendizaje móvil (FRAME, por sus siglas en inglés, Framework for the Rational Analysis for Mobile Learning) desde la consideración de tres aspectos: i) la tecnología portátil, ii) los aspectos cognitivos de los estudiantes y iii) los aspectos sociales entre los individuos. A través de la interrelación entre estos tres aspectos dicha autora intenta caracterizar el desarrollo del aprendizaje móvil como un proceso de interacción social entre los estudiantes a través del uso de recursos tecnológicos portátiles, especialmente idóneo para apoyar los procesos de enseñanza-aprendizaje a distancia.

En el presente artículo se propone el entendimiento del proceso de enseñanzaaprendizaje móvil considerando: i) la tecnología y sus particularidades físicas, ii) los estudiantes y su proceso de aprendizaje, junto con iii) los docentes y su proceso de enseñanza. Esta propuesta retoma los elementos considerados por Coll (2004) en cuanto a la trascendencia que tienen las tecnologías de la información y comunicación (TIC) como mediadoras entre estudiantes, docentes y contenidos en los procesos educativos formales. También considera la importancia de analizar las dinámicas de enseñanza-aprendizaje como formas de interacción social donde el diálogo y la comunicación entre estudiantes y docentes puede adquirir un rol protagónico en beneficio de ambos, idea propuesta inicialmente por Gordon Pask a través de la Teoría de la Conversación y posteriormente retomada por Sharples (2005) y Laurillard (2007). Finalmente se incorpora el análisis propuesto por Brown (2005) acerca del surgimiento de un nuevo paradigma en el ámbito educativo, el navegacionismo, el cual se fundamenta en la consolidación de la red como plataforma de comunicación y de difusión de grandes cantidades de información que pueden ser accedidas libremente por los usuarios, lo cual impacta de manera directa al ámbito educativo y provoca una transformación de los roles tradicionales de estudiantes y docentes. Por una parte, surge la necesidad de capacitar a los estudiantes para buscar, encontrar, identificar, manipular y evaluar la información disponible en la red, integrarla a sus labores cotidianas y compartirla con otros individuos. Por otra parte, los docentes deben propiciar acciones de comunicación y transformarse en guías/tutores en torno a cómo navegar por esta ingente cantidad de información. De esta manera, el aprendizaje en la actualidad se debe comprender como una actividad de exploración, evaluación, 
manipulación, integración y navegación por la información y conocimientos disponibles.

En el ámbito internacional, se han realizado múltiples investigaciones para explorar el potencial educativo de los recursos tecnológicos portátiles, principalmente en contextos de educación a distancia (Petit y Kukulska-Hulme, 2006; Hussain y Adeeb, 2009; Kenny, Van Neste-Kenny, Park, Burton y Meiers, 2009; Lundsford, 2010). Estos estudios destacan la necesidad de analizar más sigilosamente el uso de estos recursos por los estudiantes y docentes actuales, dada las posibilidades de acceso a la información y comunicación que propician. Por su parte, en Latinoamérica se han llevado a cabo algunos programas para incorporar su uso como herramienta didáctica de apoyo a los procesos de enseñanza-aprendizaje (UNESCO, 2012), principalmente en el marco de programas pilotos para niños y adolescentes de sectores vulnerables, programas de alfabetización para jóvenes y adultos, programas de mejoramiento de la gestión educativa, entre otros.

Cabe destacar que en el contexto mexicano se han desarrollado ciertas investigaciones acerca de los usos educativos de la tecnología portátil en educación superior, reportándose una mayor facilitación para la descarga e intercambio de información, facilidad de acceso a los contenidos de los cursos, mejor administración de los tiempos de estudio, facilitación de la comunicación entre estudiantes, fomento del trabajo en equipo, entre otras ventajas (Herrera, Lozano y Ramírez, 2008; Ramos, Herrera y Ramírez, 2010). Sin embargo, tanto los alcances como los contextos espaciotemporales de estos estudios han sido limitados (educación superior, posgrado).

Para construir una base teórica y metodológica que sustente el uso educativo de smartphones en la educación superior, primero se debe explorar y analizar la posesión de tales recursos en la comunidad universitaria, de qué manera se utilizan en las actividades académicas de estudiantes y docentes, así como su percepción acerca de la utilidad de estos recursos como herramienta pedagógica de apoyo. En el presente documento se propone una clasificación de perfiles de uso de smartphones en estudiantes y docentes de la UABC a partir de variables personales, socioeconómicas y académicas. Se espera que esta clasificación permita identificar las principales variables relacionadas con el uso de tales recursos y, sobre la base lo anterior, bosquejar su incorporación como herramienta de apoyo a las actividades académicas de ambos.

\section{Método}

\section{Contexto espacio-temporal y participantes}

La investigación se realizó en la Universidad Autónoma de Baja California (UABC), unidad Ensenada. Para el primer ciclo escolar de 2011, se contabilizaron 9,008 estudiantes, de los cuales se extrajo una muestra representativa de 1,073 estudiantes, que supone un $11.9 \%$ de la población. En el caso de los docentes, en el primer ciclo escolar de 2011 se contabilizó un total de 1,046 docentes, de los cuales se 
extrajo una muestra representativa de 249 docentes, que supone un $23.8 \%$ de la población (ver tabla I).

\begin{tabular}{cccc}
\hline & $\begin{array}{c}\text { Tamaño de } \\
\text { población }(\mathbf{N})\end{array}$ & $\begin{array}{c}\text { Tamaño de } \\
\text { muestra (n) }\end{array}$ & $\%$ \\
\hline Estudiantes & 9008 & 1073 & 11.9 \\
\hline Docentes & 1046 & 249 & 23.8 \\
\hline
\end{tabular}

Tabla I. Tamaños de población y muestra de estudiantes y docentes

Cabe destacar que para calcular la muestra representativa de estudiantes, se recurrió al algoritmo propuesto por Cuesta y Herrero (2010) que considera: tamaño de la población, valor de $Z$ correspondiente al nivel de confianza elegido $(95 \%, Z=1.96)$, frecuencia/probabilidad $(\mathrm{P})$ del factor a estudiar $(50 \%, \mathrm{P}=0.5)$ y estimación de error máximo (0.03). Este último se estableció para obtener una mayor rigurosidad en la muestra de estudiantes, pese a que la mayoría de las investigaciones sociales refieren índices de error máximo de 0.05. En el caso de los docentes, se utilizó el mismo algoritmo. Los índices utilizados fueron los mismos, a excepción de: frecuencia/probabilidad $(\mathrm{P})$ del factor a estudiar $(30 \%, \mathrm{P}=0.3)$ y estimación del error máximo $(0.05)$.

\section{Instrumento y procedimiento}

Se elaboró un instrumento de recogida de información con el propósito de analizar la posesión y uso de smartphones en estudiantes y docentes. El instrumento estuvo compuesto por las siguientes dimensiones:

- Aspectos personales. Relacionados con la información personal, situación socioeconómica, antecedentes académicos, uso de recursos tecnológicos en general, así como las estrategias y técnicas de enseñanza-aprendizaje preferidas de estudiantes y docentes.

- Aspectos tecnológicos. Referidos a la posesión de smartphones, sus características, aplicaciones y programas disponibles.

- Aspectos relacionados con el uso tecnológico. Comprenden los tipos y niveles de uso de las aplicaciones/programas ofrecidos por los smartphones, junto con la percepción de estudiantes y docentes acerca de estos recursos tecnológicos en el plano educativo y no educativo. De acuerdo con Bebell, Russell y O’Dwyer (2004), Russell, O’Dwyer, Bebell y Miranda (2004) y O’Dwyer, Russell y Bebell (2005), el uso tecnológico en general debe entenderse como un constructo multidimensional en el que se integran tipos y niveles de uso. En este caso, se aborda el constructo nivel de uso a partir del dominio y la frecuencia en la utilización de las aplicaciones/programas del smartphone. Por otro lado, se indagaron los tipos de uso a partir de la finalidad (comunicación, manejo de información y organización, destacadas como principales según Kukulska-Hulme y Traxler, 2005) de uso de tales 
recursos. Por su parte, se elaboró un nuevo constructo relacionado con el uso de smartphones, pericia de uso, siguiendo los lineamientos propuestos por Duart, Gil, Pujol y Castaño (2008) respecto al uso de recursos tecnológicos en educación superior.

En el caso de los estudiantes, el instrumento constó de 60 reactivos distribuidos de la siguiente manera: 40 ordinales, 7 categóricos, 5 dicotómicos, 4 de razón y 4 abiertos. En el caso de los docentes, el instrumento constó de 56 reactivos distribuidos en: 37 ordinales, 8 categóricos, 4 dicotómicos, 4 abiertos y 3 de razón.

La aplicación de los citados instrumentos se llevó a cabo durante el segundo semestre de 2011. En el caso de los estudiantes, se solicitó autorización a los directivos de las escuelas/facultades de la UABC y se aplicó grupalmente, seleccionando de manera aleatoria los salones de clases a partir del semestre que cada grupo llevaba cursando. En el caso de los docentes, la aplicación se ejecutó de manera personalizada, seleccionándolos aleatoriamente a partir de los porcentajes de la muestra representativa requeridos para cada campus.

\section{Análisis de datos}

Los análisis de datos se realizaron con la ayuda del paquete estadístico SPSSC, versión 19.0. Posteriormente al diseño del archivo maestro, digitalización y depuración de los datos, se obtuvieron los indicadores estadísticos descriptivos, se realizaron análisis comparativos (análisis de diferencia para muestras independientes, $t$-student) y análisis clasificatorios (K-means clusters).

\section{Resultados}

Los resultados del estudio se presentan de la siguiente manera: i) características generales, ii) posesión y uso de la tecnología y iii) clasificación de niveles de uso de smartphonesen estudiantes y docentes.

\section{Características generales de los participantes}

Las características generales de la muestra con relación al género y edad se observan en la tabla II. En estudiantes se observó una leve mayoría femenina (52.5\%) con respecto a los hombres (47.5). En docentes, se observó un equilibro ente mujeres y hombres (50.6\% y 49.4\% respectivamente). Con relación a la edad, los estudiantes arrojaron una media de 21.3 años. En cuanto a los docentes, la media de edad fue de 43.3 años.

\begin{tabular}{ccccccc}
\hline & \multicolumn{2}{c}{ Hombres } & \multicolumn{2}{c}{ Mujeres } & \multicolumn{2}{c}{ Edad } \\
\cline { 2 - 7 } & $\mathbf{n}$ & $\mathbf{\%}$ & $\mathbf{n}$ & $\mathbf{\%}$ & Media & d.e. \\
\hline Estudiantes & 509 & 47.5 & 562 & 52.5 & 21.3 & 3.8 \\
\hline Docentes & 122 & 49.4 & 125 & 50.6 & 43.3 & 10.8 \\
\hline
\end{tabular}

Tabla II. Distribución de estudiantes y docentes por género y edad ${ }^{1}$

\footnotetext{
${ }^{1}$ Nomenclatura: d.e. $=$ desviación estándar. 
En la tabla III se muestran las medias para las variables: dominio del idioma inglés y autodefinición ante el uso de recursos tecnológicos en general. Para los estudiantes, el dominio del inglés registró una media de 1.7, mientras que los docentes arrojaron una media de 2.2. Con respecto a la autodefinición ante el uso de la tecnología, los estudiantes alcanzaron una media global de 2.0. Para los docentes, la media global fue de 2.3 .

\begin{tabular}{ccccc}
\hline & \multicolumn{2}{c}{ Dominio de inglés** } & \multicolumn{2}{c}{$\begin{array}{c}\text { Autodefinición ante } \\
\text { uso de la tecnología** }\end{array}$} \\
\cline { 2 - 5 } & Media & d.e. & Media & d.e. \\
\hline Estudiantes & 1.7 & 0.8 & 2.0 & 0.6 \\
\hline Docentes & 2.2 & 0.7 & 2.3 & 0.6 \\
\hline
\end{tabular}

Tabla III. Dominio del idioma inglés y autodefinición ante la tecnología en estudiantes y docentes ${ }^{2}$

Las distribuciones de frecuencia para la variable posesión de Internet en casa se observan en la tabla IV. En estudiantes y docentes se observó un predominio de quienes poseen este recurso en su hogar. En el caso de los docentes, este predominio alcanzó el $97.2 \%$ de la muestra, mientras que en el caso de los estudiantes alcanzó el $84.2 \%$ de la muestra.

\begin{tabular}{ccccc}
\hline & \multicolumn{2}{c}{ Sin Internet } & \multicolumn{2}{c}{ Con Internet } \\
\cline { 2 - 5 } & $\mathbf{n}$ & $\mathbf{\%}$ & $\mathbf{n}$ & $\mathbf{\%}$ \\
\hline Estudiantes & 169 & 15.8 & 903 & 84.2 \\
\hline Docentes & 7 & 2.8 & 242 & 97.2 \\
\hline Tabla IV. Distribuciones de frecuencia para posesión de \\
Internet en casa en estudiantes y docentes
\end{tabular}

\section{Posesión y uso de tecnología}

El porcentaje de posesión de teléfonos celulares en la muestra alcanzó un 96.1\% en estudiantes y un $96.8 \%$ docentes, tal como se observa en la tabla V. Llama la atención que dentro de los estudiantes que poseen teléfonos celulares, un $11.5 \%$ aseveró tener más de un dispositivo. En el caso de los docentes, se observó un $8.5 \%$ que posee más de un teléfono celular.

\footnotetext{
${ }^{2}$ Nomenclatura: d.e. $=$ desviación estándar.

${ }^{*}$ Escala de Likert (0-3), donde el $\mathbf{0}=$ Nulo; $\mathbf{1}=$ Bajo; 2 =Intermedio; 3 =Avanzado.

${ }^{* *}$ Escala de Likert (0-3), donde el $\mathbf{0}=$ No familiar; $\mathbf{1}=$ Principiante; $\mathbf{2}=$ Intermedio $\mathbf{3}=$ Avanzado.
} 


\begin{tabular}{ccccccc}
\hline & \multicolumn{3}{c}{ Posesión de teléfono celular } \\
\cline { 2 - 7 } & Ninguno & \multicolumn{1}{c}{ Uno } & \multicolumn{2}{c}{ Más de 1 } \\
\cline { 2 - 7 } & $\mathbf{n}$ & $\mathbf{\%}$ & $\mathbf{n}$ & $\mathbf{\%}$ & $\mathbf{n}$ & $\mathbf{\%}$ \\
\hline Estudiantes & 42 & 3.9 & 906 & 84.6 & 123 & 11.5 \\
\hline Docentes & 8 & 3.2 & 219 & 88.3 & 21 & 8.5 \\
\hline Tabla V. Porcentaje de posesión de celular en estudiantes y \\
docentes
\end{tabular}

Para establecer la diferencia entre quienes poseen y no smartphone, se recurrió al tipo de conectividad que tienen los teléfonos celulares de los participantes (figura I). Se establecieron tres categorías: i) avanzada (conexión inalámbrica a Internet: Wi-Fi, $3 \mathrm{G} / 4 \mathrm{G}$ ); ii) básica (conexión inalámbrica a otros equipos: Bluetooth, y puertos de entrada USB); y iii) sin conectividad. Más de la mitad de los estudiantes (53.3\%) y cerca de dos tercios de los docentes $(66.1 \%)$ cuentan con un teléfono celular con conectividad avanzada. La característica fundamental de los smartphones es la convergencia de la telefonía móvil con la tecnología computacional, por lo tanto su conectividad a la red independiente del contexto espacio-temporal del usuario y, sobre la base de esto, la posibilidad de descarga de aplicaciones adicionales, el acceso a múltiples vías de comunicación y de manejo de información/medios, resulta primordial para definirlos e identificarlos. Por lo tanto, se estimó que un 53.3\% de los estudiantes $\mathrm{y}$ un $66.1 \%$ de los docentes poseen un smartphone.

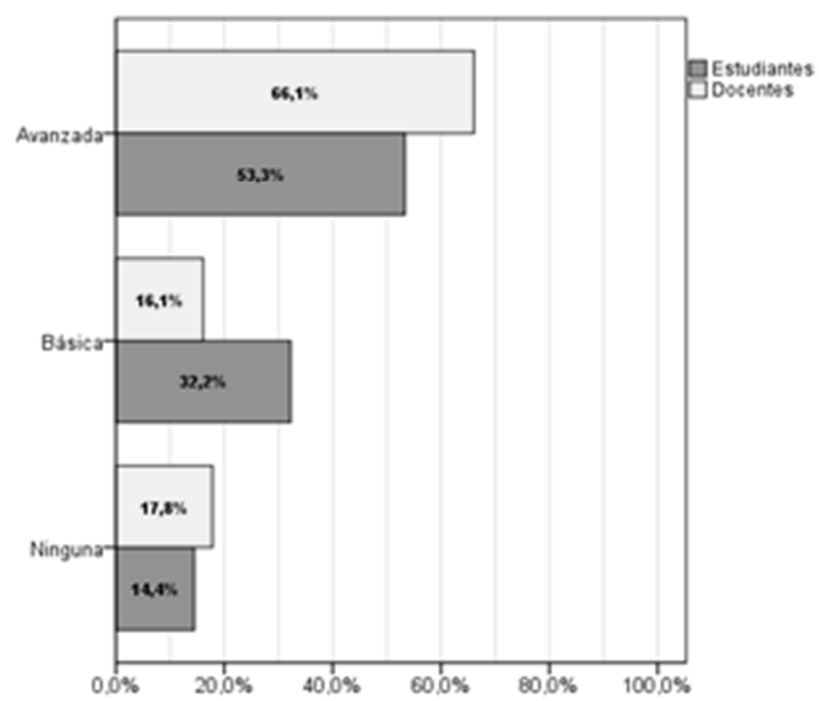

Figura I. Posesión de smartphones según el tipo de conectividad de los teléfonos celulares de los participantes 
En la tabla VI se muestran los estadísticos básicos para la variable experiencia (años) de uso del teléfono celular en general. En los estudiantes, la media global fue de 6.7 años, mientras que en los docentes la media global fue de 10.5 años.

\begin{tabular}{ccc}
\hline & \multicolumn{2}{c}{ Años de uso } \\
\cline { 2 - 3 } & Media & d.e. \\
\hline Estudiantes & 6.7 & 2.8 \\
\hline Docentes & 10.5 & 4.0 \\
\hline Tabla VI. Experiencia (años) de uso del \\
teléfono celular en estudiantes y docentes
\end{tabular}

La forma de aprender a utilizar el smartphone se estimó a través de una escala nominal de cuatro opciones en ambos participantes. Como se muestra en la tabla VII, a nivel global la mayoría de estudiantes $(88.4 \%)$ aprendió a utilizar el smartphone explorando el dispositivo por su propia cuenta. En el caso de los docentes, esta tendencia disminuye: sólo un $68.5 \%$ aprendió a usarlo bajo esta forma. La segunda manera más recurrente de aprender a utilizar el smartphone en docentes fue mediante consultas al manual o guía rápida (18.4\%).

\begin{tabular}{ccccc}
\hline Forma de aprender a usar & \multicolumn{2}{c}{ Estudiantes } & \multicolumn{2}{c}{ Docentes } \\
\cline { 2 - 5 } el smartphone & $\mathbf{n}$ & $\mathbf{\%}$ & $\mathbf{n}$ & $\mathbf{\%}$ \\
\hline Por exploración & 448 & 88.5 & 89 & 68.5 \\
\hline Consulta al manual & 40 & 7.9 & 24 & 18.4 \\
\hline Preguntas a expertos & 7 & 1.4 & 13 & 10.0 \\
\hline Consulta en Internet & 11 & 2.2 & 4 & 3.1 \\
\hline
\end{tabular}

Tabla VII. Forma de aprender a utilizar el smartphone en estudiante y docentes

Para estimar la frecuencia semanal de uso de un conjunto de aplicaciones/medios desde el smartphone, se utilizó una escala ordinal de cinco pasos (codificación 0-4). En la figura II se observan las distribuciones de la frecuencia de uso para cada una de las aplicaciones/medios en estudiantes. Las frecuencias de uso más altas fueron alcanzadas por: mensajería, llamadas de voz, reproductor de música, manejo de contactos, buscadores y navegadores de Internet, redes sociales y manejo de fotos.

\footnotetext{
${ }^{3}$ Nomenclatura: d.e. $=$ desviación estándar.
} 


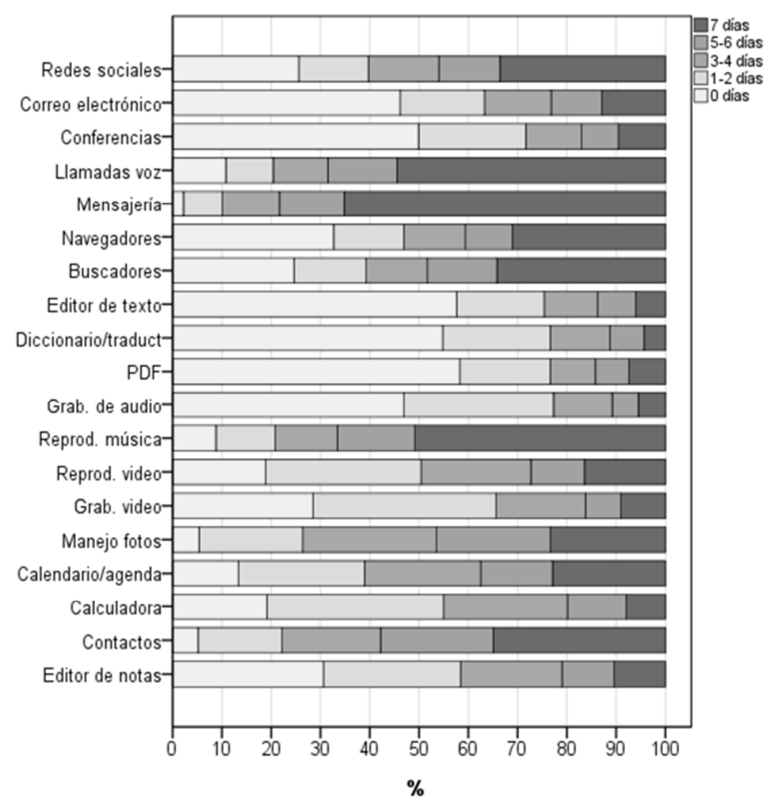

Figura II. Frecuencia de uso semanal de aplicaciones desde el smartphone en estudiantes

En el caso de los docentes, las frecuencias de uso más altas fueron alcanzadas por: llamadas de voz, mensajería, manejo de contactos, calendario/agenda, correo electrónico y navegadores de Internet (figura III).

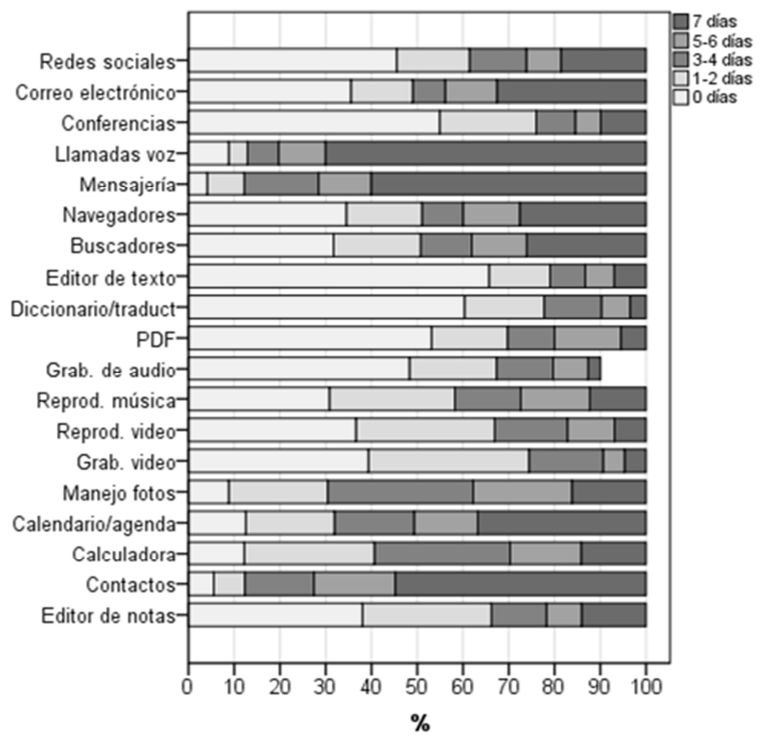

Figura III. Frecuencia de uso semanal de aplicaciones desde el smartphone en docentes 
La estimación del porcentaje de uso educativo (por ejemplo, relacionado con la realización de tareas, trabajos académicos, preparación de clases, agenda $\mathrm{u}$ organización) y no educativo (por ejemplo, juegos, reproducción de música o videos) del smartphone en ambos participantes se muestra en la tabla VIII. Los estudiantes le asignan menos de un tercio $(29.2 \%)$ del tiempo total de uso a acciones de tipo educativas. Para los docentes, el porcentaje de uso educativo del smartphone alcanzó un $34.5 \%$ del tiempo total de uso del dispositivo.

\begin{tabular}{ccc}
\hline & Uso educativo & Uso no educativo \\
\cline { 2 - 3 } & $\mathbf{\%}$ & $\mathbf{\%}$ \\
\hline Estudiantes & 29.2 & 70.8 \\
\hline Docentes & 34.6 & 65.4 \\
\hline
\end{tabular}

$\overline{\text { Tabla VIII. Porcentaje de uso educativo y uso no educativo }}$ del smartphone

Con respecto a la percepción de los participantes acerca de la utilidad del smartphone como herramienta de apoyo a sus actividades académicas, el gusto/motivación hacia su uso, así como su portabilidad/conectividad, se propusieron seis reactivos medidos con una escala de ordinal de cuatro pasos $(0=$ Totalmente en desacuerdo; 1=En desacuerdo;2=En acuerdo;3=Totalmente en acuerdo). Como se observa en la figura IV, los reactivos que obtuvieron las medias más altas fueron los referidos a la portabilidad del smartphone ("siempre cargo conmigo el celular") y al gusto por su uso ("me gusta usar el celular"). Cabe destacar que en todos los reactivos las medias más altas fueron alcanzadas por los estudiantes, a excepción del que hace referencia a la conectividad que prestan estos dispositivos ("el celular es un elemento fundamental para no estar aislado"), en el que los docentes obtuvieron una media mayor. 

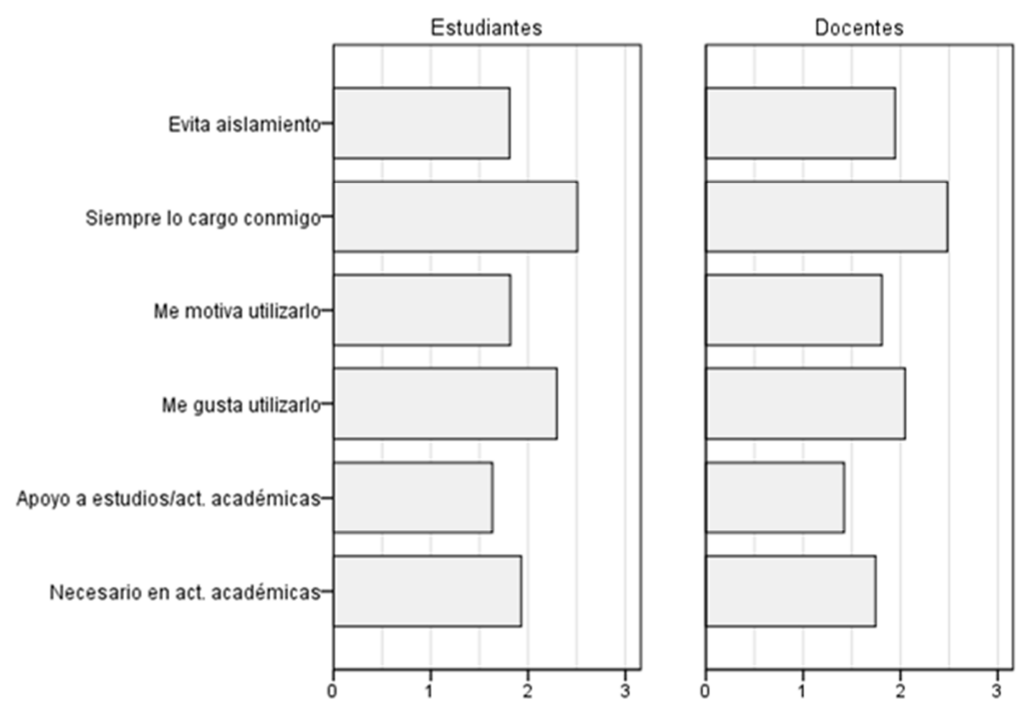

Figura IV. Percepción acerca de utilidad del smartphone para actividades académicas y extra-académicas de los participantes

De acuerdo a lo propuesto por Duart et al. (2008), la pericia de uso tecnológico se puede estimar a través de la agrupación de tres subconstructos: i) la experiencia de uso, ii) la frecuencia de uso y iii) la habilidad de uso. En el presente artículo, se retoman los planteamientos de Duart et. al (2008) para proponer un acercamiento al constructo pericia de uso de smartphones a través de: i) la frecuencia de uso, ii) la percepción acerca de su utilidad y iii) la habilidad de uso.

La frecuencia surge como resultado de la media de uso semanal de las aplicaciones/medios propuestos anteriormente (codificación 0-4). La percepción resulta de la media entre los reactivos elaborados para tal fin (codificación 0-3). Por último, para la estimación de la habilidad de uso se consideraron tres reactivos: la experiencia (años) de uso, el autoconcepto acerca del dominio de la tecnología en general (computacional, Internet, celular) y la facilidad/dificultad para aprender a utilizar el smartphone. Dado que estas variables fueron medidas con escalas distintas, se homogeneizaron de la siguiente manera: la experiencia se estandarizó y recodificó a través de una escala ordinal con codificación 0-3; la facilidad/dificultad para aprender a utilizar el celular, medida en escala ordinal, se recodificó con puntuación $0-3$ ( $0=$ Muy difícil, 1=Difícil, 2=Fácil, 3=Muy Fácil). Por último, el autoconcepto acerca del dominio tecnológico se mantuvo con escala ordinal, codificación 0-3. Por lo tanto, la habilidad de uso quedó determinada a través de una escala ordinal, codificación 0-3.

De esta manera, la pericia de uso de smartphones resulta de la convergencia entre la frecuencia de uso, la habilidad en su manejo y la percepción que los participantes tienen acerca de su utilidad tanto en sus actividades académicas como extraacadémicas. Considerando las codificaciones de cada variable, los valores que adopta 
la pericia de uso de la tecnología fluctúan entre 0 y 10 . En la tabla IX se muestran las medias de esta variable para ambos participantes. Los estudiantes arrojaron una media de pericia más alta que los docentes, aunque no se observaron diferencias estadísticamente significativas al respecto (al nivel $\mathrm{p}<0.05$ ).

\begin{tabular}{cccccc}
\hline & \multicolumn{5}{c}{ Pericia de uso de smartphones } \\
\cline { 2 - 5 } & $\mathbf{n}$ & Media & d.e. & t & Sig. \\
\hline Estudiantes & 486 & 6.0 & 1.2 & \multirow{2}{*}{1.81} & .07 \\
\hline Docentes & 121 & 5.8 & 1.4 & & \\
\hline
\end{tabular}

Tabla IX. Análisis de diferencia para muestras independientes ( $t$-student) entre estudiantes y docentes en torno a la pericia de uso de smartphones

\section{Clasificación de niveles de uso de smartphones}

Para establecer la clasificación de perfiles de uso de smartphones en estudiantes y docentes, se ejecutó un análisis de conglomerados k-medias (K-Means Clusters) con base a cuatro variables relacionadas con el uso de smartphones: i) facilidad/dificultad para aprender a usarlo, ii) experiencia de uso, iii) media de frecuencia semanal de uso y iv) media de percepción acerca de su utilidad. El objetivo fue obtener ciertos perfiles de uso de smartphones en estudiantes y docentes de la UABC y caracterizarlos a partir de variables personales, socioeconómicas y académicas.

\section{Clasificación de estudiantes}

La contribución de cada variable a la clasificación de conglomerados se muestra en la tabla X. El análisis ANOVA sugiere que la variable con mayor trascendencia dentro de la clasificación fue la experiencia de uso, seguida de la media de frecuencia semanal de uso y la facilidad/dificultad para aprender a utilizar el smartphone.

\begin{tabular}{|c|c|c|c|c|c|c|}
\hline & \multicolumn{2}{|c|}{ Conglomerados } & \multicolumn{2}{|c|}{ Error } & \multirow[b]{2}{*}{$\mathbf{F}$} & \multirow[b]{2}{*}{ Sig. } \\
\hline & $\begin{array}{c}\text { Media } \\
\text { cuadrática }\end{array}$ & $\begin{array}{c}\text { Grados de } \\
\text { libertad }\end{array}$ & $\begin{array}{c}\text { Media } \\
\text { cuadrática }\end{array}$ & $\begin{array}{c}\text { Grados de } \\
\text { libertad }\end{array}$ & & \\
\hline $\begin{array}{l}\text { Facilidad/dificultad para aprender } \\
\text { a usar smartphone }\end{array}$ & 29.0 & 1 & 0.4 & 486 & 67.0 & .00 \\
\hline $\begin{array}{l}\text { Experiencia (años) de uso de } \\
\text { smartphone }\end{array}$ & 143.0 & 1 & 0.5 & 486 & 304.4 & .00 \\
\hline $\begin{array}{l}\text { Frecuencia media semanal de } \\
\text { uso }\end{array}$ & 69.2 & 1 & 0.4 & 486 & 171.4 & .00 \\
\hline $\begin{array}{l}\text { Percepción acerca de utilidad de } \\
\text { smartphone }\end{array}$ & 18.7 & 1 & 0.2 & 486 & 76.8 & .00 \\
\hline
\end{tabular}

Tabla X. Tabla ANOVA de variables relacionadas con de uso de smartphones en estudiantes

A partir del valor de los centroides (centros multivariantes) de cada variable, se identificaron dos perfiles de uso de smartphones: i) bajo y ii) avanzado (figura XI). Un $58.0 \%$ de los estudiantes se aglomeró en el perfil de uso avanzado, mientras un $42.0 \%$ se ubicó en el perfil de uso bajo. 


\begin{tabular}{|c|c|c|}
\hline & \multicolumn{2}{|c|}{ Conglomerados } \\
\hline & Bajo & Avanzado \\
\hline Facilidad/dificultad para aprender a usar celular* & 2 & 3 \\
\hline Experiencia (años) de uso de celular & 1 & 2 \\
\hline Media frecuencia semanal de uso** & 1.4 & 2.1 \\
\hline Media percepción acerca de utilidad de celular*** & 1.9 & 2.3 \\
\hline Número de casos & 205 & 283 \\
\hline Porcentaje & 42.0 & 58.0 \\
\hline
\end{tabular}

Tabla XI. Centroides finales de los conglomerados para cada variable relacionada con el uso de smartphones en estudiantes ${ }^{4}$

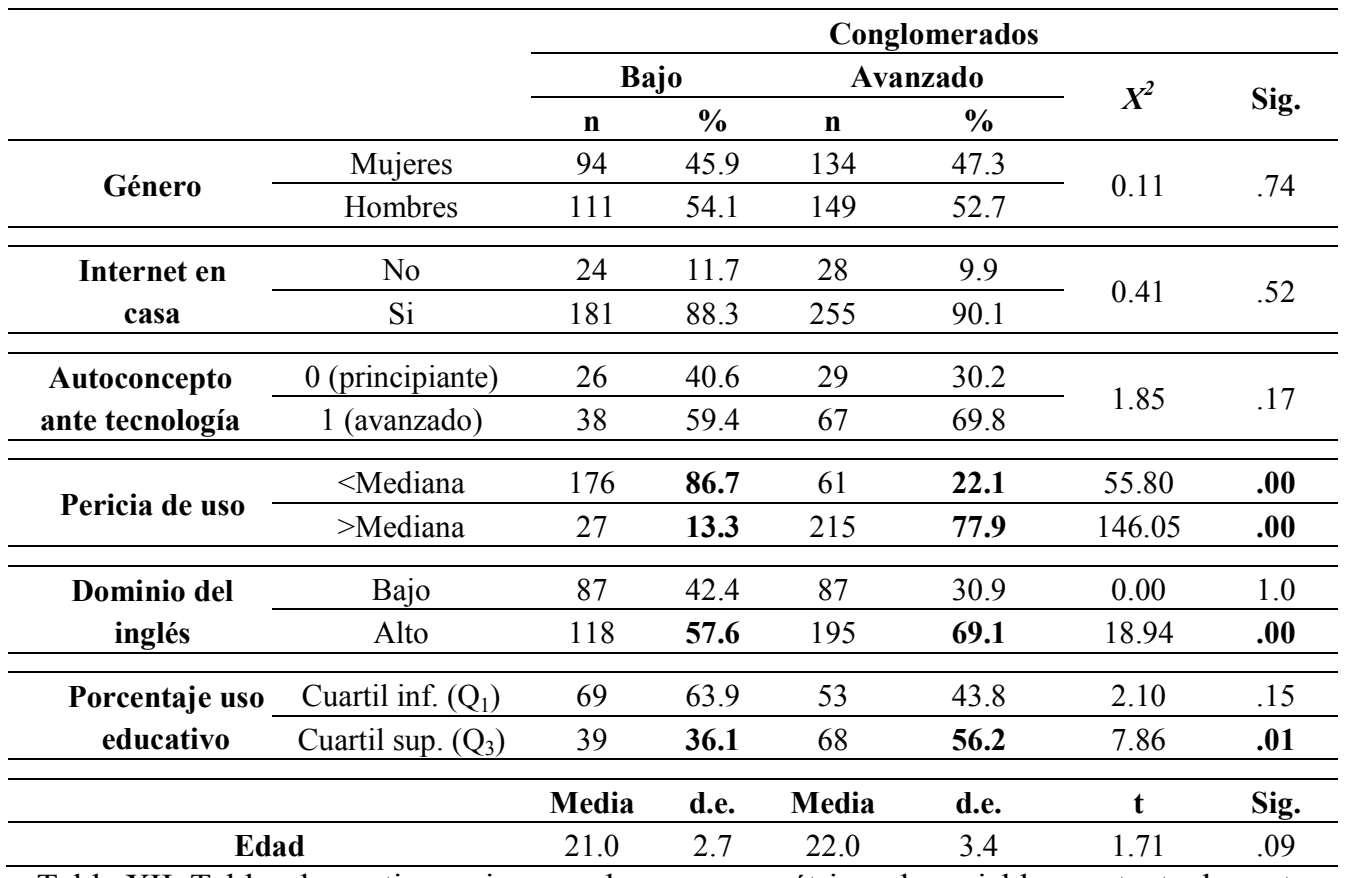

Tabla XII. Tablas de contingencia y pruebas no paramétricas de variables contextuales entre conglomerados de uso de smartphones en estudiantes ${ }^{5}$

4 * Escala de Likert (0-3); donde 0=Muy difícil; 1=Difícil; 2=Fácil; $3=$ =Muy fácil

** Escala de Likert (0-4): donde $\mathbf{0}=$ Ningún día...4= todos los días de la semana

*** Escala de Likert (0-3): donde $\mathbf{0}=$ totalmente en desacuerdo... $\mathbf{3}=$ totalmente en acuerdo).

${ }^{5}$ Nomenclatura: d.e. $=$ desviación estándar. 
En la tabla XII se muestran las tablas de contingencia de las distribuciones de frecuencia de un conjunto de variables contextuales entre los perfiles de uso de smartphones en estudiantes. Se hicieron pruebas no paramétricas (Chi-cuadrado de Pearson) para analizar la significatividad de las diferencias de distribución de cada variable contextual en ambos conglomerados, además de un análisis de diferencia $(t-$ student para muestras independientes) para la variable edad. Para cada variable contextual se establecieron grupos de contraste. Como se observa, las variables pericia de uso, dominio del inglés y porcentaje de uso educativo asignado al smartphone arrojaron diferencias significativas (al nivel $\mathrm{p}<0.05$ ) en sus distribuciones de frecuencia entre los perfiles de uso. Esto implica que en el conglomerado de uso avanzado se ubican aquellos estudiantes con un mayor nivel de pericia de uso ( $>$ Mediana), con un dominio alto del idioma inglés y quienes le asignan un porcentaje de uso educativo significativamente mayor (cuartil superior, $\mathrm{Q}_{3}$ ) al smartphone.

Sobre la base de lo anterior, a continuación se presenta el perfil de uso de cada conglomerado:

- Conglomerado de uso bajo. Este grupo concentró a menos de la mitad $(42.0 \%)$ de los estudiantes que poseen smartphones y arrojó una media de pericia de uso de igual a 5.0 (dentro de una escala de 0-10). Los centroides de las variables relacionadas con el uso de smartphones fueron los más bajos entre los dos conglomerados. Con respecto al género, se observó una leve mayoría de hombres (54.1\%) y a partir de la edad, se observó una media de 21 años.

Por su parte, se observó un predominio de estudiantes que poseen el recurso de Internet en su hogar ( $88.3 \%$ de estudiantes). Respecto al dominio del idioma inglés, más de la mitad de los estudiantes (57.6\%) se ubicaron en un nivel alto. Esto contrastó significativamente con el conglomerado de uso avanzado, donde un $69.1 \%$ de los estudiantes se ubicó en un nivel de dominio alto de este idioma.

El autoconcepto de los estudiantes frente al uso de la tecnología en general mostró un predominio de quienes se autoevalúan en un nivel avanzado (59.4\%). Sin embargo, considerando la pericia de uso de smartphones, se observó un porcentaje mayoritario de estudiantes (86.7\%) ubicados bajo la mediana estadística $\left(\mathrm{Q}_{2}\right)$ de dicha variable. Esto contrasta significativamente con el conglomerado de uso avanzado, donde sólo un $22.1 \%$ de los estudiantes se ubicó bajo $\mathrm{Q}_{2}$ de pericia de uso. Por último, cerca de dos tercios $(63.9 \%)$ de los estudiantes de este conglomerado no asigna ningún tipo de uso educativo a su teléfono inteligente, frente a un $36.1 \%$ que sí lo utiliza en el marco de sus actividades académicas (trabajos, tareas, etc.).

- Conglomerado de uso avanzado. Por su parte, el grupo de uso avanzado de smartphones concentró el mayor porcentaje de estudiantes (58.0\%), junto con la media más alta de la variable pericia de uso de tales recursos (media $=6.7$ ). A su vez, los centroides de las variables asociadas a la clasificación de perfiles de uso de smartphones alcanzaron sus valores más altos en este conglomerado. 
Respecto al género, continúa la tendencia mostrada en el grupo anterior, observándose un leve predominio de hombres (52.7\%) en comparación con mujeres (47.3\%). En cuanto a la edad, en este conglomerado arrojó una media de 22 años, levemente mayor al conglomerado de uso bajo.

Con relación al dominio del idioma inglés, más de dos tercios (69.1\%) de los estudiantes se ubicaron en un nivel alto. Paralelamente, dentro de este conglomerado se acentúa la tendencia observada en el grupo anterior respecto a la posesión de Internet en casa: un $90.1 \%$ de los estudiantes poseen este recurso, frente a un $9.9 \%$ que no tienen acceso a Internet en su hogar.

Considerando el autoconcepto ante el uso de los recursos tecnológicos en general, se observó un incremento del predominio de estudiantes que se autoevalúan en un nivel avanzado, con un $69.8 \%$. A su vez, más de tres cuatros $(77.9 \%)$ de los estudiantes que poseen un smartphone se ubicaron sobre la mediana estadística $\left(\mathrm{Q}_{2}\right)$ de pericia de uso, lo que contrasta significativamente con el conglomerado de uso bajo, donde sólo un $13.3 \%$ se posicionó sobre $\mathrm{Q}_{2}$. Por último, se observó un predominio de estudiantes que asignan un mayor porcentaje de uso educativo al smartphone $\left(56.2 \%\right.$ del $\mathrm{Q}_{3}$ de esta variable), lo que contrastó de manera significativa con el conglomerado de uso bajo, donde un $36.1 \%$ de los estudiantes se ubicaron dentro del $\mathrm{Q}_{3}$ de esta variable.

En la tabla XIII se presenta un resumen del perfil de cada conglomerado de uso de smartphones en estudiantes a partir de las tendencias de las variables contextuales:

\begin{tabular}{lll}
\hline \multirow{2}{*}{ VARIABLES } & \multicolumn{2}{c}{ Conglomerados de uso de smartphones } \\
\cline { 2 - 3 } & \multicolumn{1}{c}{ Bajo } & \multicolumn{1}{c}{ Avanzado } \\
\hline Género & Predominio hombres $(54.1 \%)$ & Predominio hombres $(52.7 \%)$ \\
\hline Edad & Media=21 años & Media=22 años \\
\hline Internet en casa & Predominio SI tienen $(88.3 \%)$ & Predominio SI tienen $(90.1 \%)$ \\
\hline Autoconcepto tec. & Predominio avanzado $(59.4 \%)$ & Predominio avanzado $(69.8 \%)$ \\
\hline Pericia de uso & Predominio baja $\left(<\mathrm{Q}_{2}, 86.7 \%\right)$ & Predominio alta $\left(>\mathrm{Q}_{2}, 77.9 \%\right)$ \\
\hline Dominio del inglés & Predominio alto $(57.6 \%)$ & Predominio alto $(69.1 \%)$ \\
\hline \% uso educativo & Predominio bajo $\left(\mathrm{Q}_{1}: 63.9 \%\right)$ & Predominio alto $\left(\mathrm{Q}_{3}: 56.2 \%\right)$ \\
\hline
\end{tabular}

Tabla XIII. Resumen del perfil de conglomerados de niveles de uso de smartphones en estudiantes.

\section{Clasificación de docentes}

En la tabla XIV se muestra la contribución de cada variable a la clasificación de perfiles de uso de smartphones en docentes. A través del análisis ANOVA se observa que la variable con mayor trascendencia fue la experiencia de uso, seguida de la media de frecuencia semanal de uso y la facilidad/dificultad para aprender a utilizar el dispositivo. Esta tendencia se observó de la misma manera en el caso de los estudiantes. 


\begin{tabular}{|c|c|c|c|c|c|c|}
\hline & \multicolumn{2}{|c|}{ Conglomerados } & \multicolumn{2}{|c|}{ Error } & \multirow[b]{2}{*}{$\mathbf{F}$} & \multirow[b]{2}{*}{ Sig. } \\
\hline & $\begin{array}{c}\text { Media } \\
\text { cuadrática }\end{array}$ & $\begin{array}{c}\text { Grados de } \\
\text { libertad }\end{array}$ & $\begin{array}{c}\text { Media } \\
\text { cuadrática }\end{array}$ & $\begin{array}{l}\text { Grados de } \\
\text { libertad }\end{array}$ & & \\
\hline $\begin{array}{l}\text { Facilidad/dificultad para } \\
\text { aprender usar celular }\end{array}$ & 2.8 & 1 & 0.5 & 119 & 5.4 & .02 \\
\hline $\begin{array}{l}\text { Experiencia (años) de uso de } \\
\text { celular }\end{array}$ & 35.3 & 1 & 0.5 & 119 & 70.3 & .00 \\
\hline $\begin{array}{l}\text { Media frecuencia semanal de } \\
\text { uso }\end{array}$ & 29.8 & 1 & 0.4 & 119 & 78.1 & .00 \\
\hline $\begin{array}{l}\text { Media percepción acerca de } \\
\text { utilidad de celular }\end{array}$ & 6.9 & 1 & 0.2 & 119 & 36.4 & .00 \\
\hline
\end{tabular}

Tabla XIV. Tabla ANOVA de variables relacionadas con el uso de smartphones en docentes

A través del análisis de conglomerados k-medias (K-Means Clusters) se establecieron dos perfiles de uso de smartphones en docentes, tal como se observa en la tabla XV: i) bajo y ii) avanzado. Cabe destacar que el grupo de uso bajo agrupó a un mayor porcentaje $(52.1 \%)$ de docentes en comparación con el grupo de uso avanzado de smartphones.

\begin{tabular}{|c|c|c|}
\hline & \multicolumn{2}{|c|}{ Conglomerados } \\
\hline & Bajo & Avanzado \\
\hline Facilidad/dificultad para aprender a usar celular* & 2.1 & 2.4 \\
\hline Experiencia (años) de uso de celular & 1.0 & 2.0 \\
\hline Media frecuencia semanal de uso** & 1.1 & 2.1 \\
\hline Media percepción acerca de utilidad de celular*** & 1.8 & 2.3 \\
\hline Número de casos & 63 & 58 \\
\hline Porcentaje & 52.1 & 47.9 \\
\hline
\end{tabular}

Tabla XV. Centroides finales de los conglomerados para cada variable relacionada con el uso de smartphones en docentes ${ }^{6}$

En la tabla XVI se muestran las distribuciones de frecuencia de un conjunto de variables contextuales entre los conglomerados de uso de smartphones, las pruebas paramétricas ( $t$-student para muestras independientes, en el caso de la variable edad) y no paramétricas (Chi-cuadrado de Pearson) para analizar la significatividad de las diferencias de distribución de cada variable contextual entre los conglomerados propuestos. Tal como en el caso de los estudiantes, se observa que la pericia de uso, el dominio del idioma inglés y el porcentaje de uso educativo asignado al smartphone arrojaron diferencias significativas en sus distribuciones de frecuencia entre los conglomerados propuestos. Por lo tanto, el conglomerado de uso avanzado quedó compuesto por aquellos docentes con una mayor pericia de uso de los smartphones (> Mediana), con un alto dominio del idioma inglés y que le asignan un uso educativo significativamente mayor a este recurso tecnológico (> Mediana).

\footnotetext{
6 * Escala de Likert (0-3); donde 0=Muy difícil; $\mathbf{1}=$ Difícil; $2=$ =Fácil; $3=$ Muy fácil

**Escala de Likert (0-4); donde $\mathbf{0}=$ Ningún día...4= todos los días de la semana

$* * *$ Escala de Likert (0-3); donde $\mathbf{0}=$ totalmente en desacuerdo... $3=$ totalmente en acuerdo).
} 


\begin{tabular}{|c|c|c|c|c|c|c|c|}
\hline & & \multicolumn{6}{|c|}{ Conglomerados } \\
\hline & & \multicolumn{2}{|c|}{ Bajo } & \multicolumn{2}{|c|}{ Avanzado } & \multirow{2}{*}{$X^{2}$} & \multirow{2}{*}{ Sig. } \\
\hline & & $\mathbf{n}$ & $\%$ & $\mathbf{n}$ & $\%$ & & \\
\hline \multirow{2}{*}{ Género* } & Mujeres & 31 & 49.2 & 27 & 46.6 & \multirow{2}{*}{.09} & \multirow{2}{*}{.77} \\
\hline & Hombres & 32 & 50.8 & 31 & 53.4 & & \\
\hline \multirow{2}{*}{$\begin{array}{c}\text { Internet en } \\
\text { casa }\end{array}$} & No & 3 & 4.8 & 1 & 1.7 & \multirow{2}{*}{.87} & \multirow{2}{*}{.35} \\
\hline & $\mathrm{Si}$ & 60 & 95.2 & 57 & 98.3 & & \\
\hline \multirow{2}{*}{$\begin{array}{l}\text { Autoconcepto ante } \\
\text { tecnología** }\end{array}$} & $<$ Mediana & 41 & 65.1 & 26 & 44.8 & 3.36 & .07 \\
\hline & $>$ Mediana & 22 & 34.9 & 32 & 55.2 & 1.85 & .17 \\
\hline \multirow{2}{*}{$\begin{array}{c}\text { Pericia de } \\
\text { uso** }\end{array}$} & $<$ Mediana & 54 & 85.7 & 6 & 10.5 & 38.40 & .00 \\
\hline & $>$ Mediana & 9 & 14.3 & 51 & 89.5 & 29.40 & .00 \\
\hline \multirow{2}{*}{$\begin{array}{c}\text { Dominio del } \\
\text { inglés* }\end{array}$} & Bajo & 11 & 17.5 & 2 & 3.4 & 6.23 & .01 \\
\hline & Alto & 52 & 82.5 & 56 & 96.6 & 0.15 & .70 \\
\hline \multirow{2}{*}{$\begin{array}{c}\text { Porcentaje uso } \\
\text { educativo** }\end{array}$} & $<$ Mediana & 39 & 61.9 & 17 & 29.3 & 8.64 & .00 \\
\hline & $>$ Mediana & 24 & 38.1 & 41 & 70.7 & 4.45 & .04 \\
\hline & & Media & d.e. & Media & d.e. & $\mathbf{t}$ & Sig. \\
\hline \multicolumn{2}{|c|}{ Edad } & 40.5 & 9.4 & 40.0 & 9.8 & .26 & .80 \\
\hline
\end{tabular}

Tabla XVI. Tablas de contingencia y pruebas no paramétricas de variables contextuales entre conglomerados de uso de smartphones en docentes ${ }^{7}$

En base a lo anterior se presenta el perfil de cada conglomerado:

- Conglomerado de uso bajo. Este grupo concentró el porcentaje mayor de docentes $(52.1 \%)$ y mostró la media más baja de pericia de uso (media=4.8). De la misma manera, los centroides de las variables relacionadas con los perfiles de uso de smartphones fueron los más bajos entre los conglomerados. Se observó una distribución equilibrada entre hombres (50.8\%) y mujeres $(49.2 \%)$ a partir de la variable género. La media de la variable edad fue de 40.5 años.

Por su parte, se observó un predominio de docentes que poseen el recurso de Internet en su hogar $(95.2 \%)$. Con relación al manejo del idioma inglés, este conglomerado concentró un $17.5 \%$ de docentes que tienen un dominio bajo. Se observaron diferencias significativas con respecto al conglomerado de uso avanzado, donde sólo un $3.4 \%$ de docentes se ubicó dentro de un nivel de dominio bajo de este idioma.

El autoconcepto de los docentes acerca del dominio de los recursos tecnológicos en general arrojó cerca de dos tercios (65.1\%) bajo la mediana estadística de esta variable, lo que implica un manejo limitado de recursos

${ }^{7}$ Nomenclatura: d.e.=desviación estándar. 
computacionales, Internet y portátiles. A su vez, una proporción similar de docentes no le asigna un uso mayormente educativo a su dispositivo: un $61.9 \%$ de docentes se ubicó bajo $\mathrm{Q}_{2}$ de la variable porcentaje de uso educativo asignado al smartphone. Esto contrasta significativamente con el conglomerado de uso avanzado, donde sólo un 29.3\% de docentes se ubicó bajo $\mathrm{Q}_{2}$ de esta variable. Con respecto a la pericia de uso, se observó una tendencia análoga: el $85.7 \%$ de los docentes se ubicó bajo la mediana de dicha variable, lo que representó una diferencia estadísticamente significativa con el conglomerado de uso avanzado, dónde sólo un $10.5 \%$ de docentes se ubicó bajo $\mathrm{Q}_{2}$ de pericia de uso.

- Conglomerado de uso avanzado. El grupo de uso avanzado de smartphones concentró un menor porcentaje de docentes $(47.9 \%)$ y una media mayor de la variable pericia de uso (media=6.9). A su vez, los centroides de las variables asociadas a los perfiles de uso de smartphones alcanzaron sus valores más altos en este conglomerado.

Respecto al género, se acentuó el predominio de hombres (53.4\%) por sobre las mujeres $(46.6 \%)$. En cuanto a la edad, la media se mantuvo con poca variación (40 años) respecto al conglomerado de uso bajo.

El predominio de docentes que posee conexión a Internet en su hogar se incrementó en este conglomerado: un 98.3\% cuenta con este recurso. Por su parte, se observó la misma tendencia para el dominio del inglés: el predominio de docentes $(96.6 \%)$ que tiene un manejo alto de este idioma se acentuó con respecto al conglomerado de uso bajo $(82.5 \%)$.

Respecto al autoconcepto frente al uso de recursos tecnológicos en general, más de la mitad (55.2\%) de los docentes se ubicó sobre la media estadística de dicha variable. La pericia de uso mostró tendencias claras: el $89.5 \%$ de los docentes se ubicó sobre $\mathrm{Q}_{2}$ de esta variable, lo cual contrastó significativamente con el conglomerado de uso bajo, donde sólo un $14.3 \%$ de docentes se ubicó sobre $\mathrm{Q}_{2}$ de pericia de uso. Por su parte, más de dos tercios $(70.7 \%)$ de los docentes de este conglomerado asignan un uso eminentemente educativo al smartphone, lo que arrojó diferencias estadísticamente significativas respecto al conglomerado de uso bajo, donde sólo un $38.1 \%$ de docentes se ubicó sobre la mediana estadística $\left(\mathrm{Q}_{2}\right)$ de esta variable.

En la tabla XVII se presenta un resumen del perfil de cada conglomerado de uso de smartphones en docentes a partir de las tendencias de las variables contextuales: 


\begin{tabular}{lll}
\hline \multirow{2}{*}{ VARIABLES } & \multicolumn{2}{c}{ Conglomerados de uso de smartphones } \\
\cline { 2 - 3 } & \multicolumn{1}{c}{ Bajo } & \multicolumn{1}{c}{ Avanzado } \\
\hline Género & Predominio hombres $(50.8 \%)$ & Predominio hombres $(53.4 \%)$ \\
\hline Edad & Media $=40.5$ años & Media $=40$ años \\
\hline Internet en casa & Predominio SI tienen $(95.2 \%)$ & Predominio SI tienen $(98.3 \%)$ \\
\hline Autoconcepto tec. & Predominio bajo $\left(<\mathrm{Q}_{2}, 65.1 \%\right)$ & Predominio avanzado $\left(>\mathrm{Q}_{2}, 55.2 \%\right)$ \\
\hline Pericia de uso & Predominio baja $\left(<\mathrm{Q}_{2}, 85.7 \%\right)$ & Predominio alta $\left(>\mathrm{Q}_{2}, 98.5 \%\right)$ \\
\hline Dominio del inglés & Predominio alto $(82.5 \%)$ & Predominio alto $(96.6 \%)$ \\
\hline$\%$ uso educativo & Predominio bajo $\left(<\mathrm{Q}_{2}, 61.9 \%\right)$ & Predominio alto $\left(>\mathrm{Q}_{2}, 70.7 \%\right)$ \\
\hline Tabla XVII.
\end{tabular}

Tabla XVII. Resumen del perfil de conglomerados de niveles de uso de smartphones en docentes.

\section{Conclusiones}

La posesión y uso de recursos tecnológicos portátiles en general, y de smartphones en particular, es un fenómeno que avanza con una velocidad irrefrenable en la sociedad actual. Ante esto, surge la necesidad de explorar de qué manera están utilizando estos recursos tanto estudiantes como docentes y cuáles son los caminos idóneos para utilizarlos en beneficio de sus actividades académicas.

A partir de la muestra representativa de una universidad pública de México (UABC), se observó que un alto porcentaje de docentes $(66.1 \%)$ y estudiantes $(53.3 \%)$ poseen y utilizan smartphones. Pese a que los porcentajes de uso educativo de estos recursos (relacionados con la realización de tareas, trabajos, preparación de clases, organización) bordean un tercio del tiempo total, esta tendencia invita a reflexionar acerca de la rapidez con que van penetrando en las actividades académicas de ambos actores y, por lo tanto, acerca de su potencial educativo.

El análisis de las aplicaciones/medios con mayor frecuencia de uso en estudiantes y docentes sugiere nuevas formas de interacción social y nuevas formas de interactividad con cuerpos de información a través del uso de smartphones. En efecto, el uso de mensajería instantánea (SMS), llamadas de voz, redes sociales (en el caso de estudiantes) y correo electrónico (en el caso de docentes) desde el smartphone, indica la apertura de los canales de comunicación y las posibilidades para desarrollar dinámicas de enseñanza-aprendizaje basadas en la construcción y exploración del conocimiento, el control del propio aprendizaje, el trabajo conjunto, la realización de proyectos, la conversación y la colaboración entre estudiantes y docentes (KukulskaHulme y Traxler, 2005; Laurillard, 2007). A su vez, la utilización de buscadores y navegadores de Internet, manejo de fotos, reproductor de música, calendario y agenda desde el smartphone, sugiere el desarrollo de nuevas maneras de acceder y manipular la información y medios, lo cual representa grandes desafíos a los procesos enseñanzaaprendizaje formales y a las maneras en que tradicionalmente se han manejado y transmitido los contenidos educativos.

A su vez, la clasificación de perfiles de uso de smartphones en estudiantes y docentes sugiere que los grupos de uso avanzado se identificaron con un mayor autoconcepto ante el dominio de la tecnología en general (celular, computacional, 
Internet), con un mayor tiempo de uso de estos recursos asignado a labores académicas y con una mayor pericia de uso al respecto, lo que implica una mayor frecuencia, percepción y habilidad (experiencia y dominio) en su manejo. De esta manera, los perfiles de uso avanzado de smartphones en estudiantes y docentes se caracterizaron por aglomerar a aquellos individuos con mayores índices de uso educativo de tales recursos, así como también mayores niveles de dominio de la tecnología en general (en el caso de los docentes). Cabe destacar que la variable dominio del idioma inglés arrojó diferencias significativas en ambos actores a favor del conglomerado de uso avanzado de los smartphones, donde se concentró un mayor porcentaje de participantes que manejan de mejor manera este idioma.

Traxler (2007 y 2009) reporta que si bien desde algunas perspectivas se ha tratado de comprender el aprendizaje móvil como una simple evolución del aprendizaje apoyado por recursos electrónicos (e-Learning), el desarrollo teórico y metodológico al respecto debe intentar esclarecer las diferencias fundamentales entre el aprendizaje móvil y otras formas de aprendizaje. En este sentido, como lo destaca Walker (2007), el aprendizaje móvil no se debe reducir sólo a la entrega de contenidos a través de pequeñas pantallas. Se trata más bien de reconsiderar y repensar los procesos de enseñanza-aprendizaje dentro de una sociedad en la que los individuos se encuentran en constante movimiento, surgiendo múltiples contextos idóneos para enseñar y aprender.

Algunas investigaciones en torno al uso de los smartphones como herramienta de apoyo a los procesos educativos (Hussain y Adeeb, 2009; Kenny et al. 2009; Kukulska-Hulme y Traxler, 2005; UNESCO, 2012) destacan que estos recursos tecnológicos pueden ser de gran utilidad para estudiantes y docentes. Esta utilidad remite tanto al acceso a la información y comunicación, como al manejo de medios y aspectos organizacionales que estos recursos propician.

A partir de las aplicaciones ofrecidas por los smartphones y de las formas de uso reportadas por estudiantes y docentes de la UABC, en el presente artículo se destacan cuatro aristas desde las cuales se puede enfocar el uso educativo de estos recursos tecnológicos. Primero, el acceso ubicuo a la información y conocimientos, lo cual permite a los docentes y estudiantes tener disponibilidad inmediata a cuerpos de información relacionados con sus materias. A su vez, esto permite acceder a la información en el marco de actividades académicas tanto fuera del salón de clases (tareas en el marco de salidas a terreno, trabajos de campo, visitas a museos, centros de salud, entre otros) como dentro del aula (actividades grupales que requieren búsqueda de información inmediata). Segundo, la comunicación constante y a través de múltiples vías entre estudiantes y docentes. A partir de la interacción social por medio de los smartphones se propicia el desarrollo de actividades grupales colaborativas en las cuales los estudiantes pueden estar intercambiando información, opiniones y puntos de vista (por medio de foros asincrónicos, chats y creación de grupos de trabajo en redes sociales), mientras que los docentes pueden retroalimentar y monitorear los avances de los estudiantes a través de su tutoría. Tercero, la multiplicidad de medios reunidos en los smartphones (manejo de voz, video, imágenes, fotos) permite el desarrollo de nuevas formas de almacenar, procesar y compartir la información, ya que posibilita a 
los estudiantes capturar a través de fotografías, videos y grabación de voz los contenidos expuestos por el docente en clases, así como capturar información relevante fuera del aula y posteriormente compartirla con compañeros y docentes. A su vez, permite a los docentes almacenar recursos y contenidos educativos en distintos formatos (texto, fotos, videos, audio) y transportarlos al aula sin mayores inconvenientes. Por último, la cuarta arista se refiere a aspectos organizacionales de estudiantes y docentes relacionados con sus actividades académicas. Esto implica el uso de la agenda electrónica o block de notas para tomar apuntes, el calendario para recordar fechas importantes (entrega de tareas, trabajos, exámenes) y el almacenamiento de contactos en el marco de actividades académicas.

El presente artículo propone una clasificación de perfiles de uso de smartphones en estudiantes y docentes a partir de ciertas variables personales, socioeconómicas y académicas A partir de esta clasificación se espera aportar elementos para una mejor comprensión en torno al uso de estos recursos y su implementación como herramienta de apoyo a los procesos de enseñanza-aprendizaje en el nivel educativo superior. Sin embargo, queda pendiente la exploración del impacto que tiene la posesión y uso de estos recursos en variables de corte académico, especialmente en la determinación del rendimiento académico. Si bien algunos estudios ya han explorado de qué manera el uso de la tecnología impacta en variables de este tipo (Duart et al. 2008), aún no se ha profundizado en el impacto que la tecnología portátil podría tener en variables de corte académico y, por lo tanto, si eventualmente podría considerarse un factor determinante al respecto, dada su posesión y uso irrefrenables en la actualidad y las perspectivas de su incremento en el futuro.

\section{Referencias bibliográficas}

BEBELL, D., RUSSELL, M. Y O'DWYER, L. (2004). Measuring teachers' technology uses: Why multiple-measures are more revealing. Journal of Research on Technology in Education, 37 (1), 45-63.

BRAZUELO, F. Y CACHEIRO, M.L. (2010). Diseño de páginas web educativas para teléfonos móviles. Edutec, revista Electrónica de Investigación Educativa, 32. Recuperado el 25 de mayo de 2012 en: http://edutec.rediris.es/ Revelec2/revelec32/articulos_n32_pdf/Edutec-e_n32_Brazuelo_Cacheiro.pdf

BROWN, T. (2005). Beyond constructivism: Exploring future learning paradigms. Education Today (2), Aries Publishing Company, Thames, New Zeland. Recuperado el 8 de marzo de 2009 en: http://pedagogy.ir/images/pdf/beyond_constructivism.pdf

CABERO, J. (2003). Tecnología educativa. Diseño y utilización de medios en la enseñanza. Barcelona: Paidós.

COLL, C. (2004). Psicología de la educación y prácticas educativas mediadas por las tecnologías de la información y comunicación. Una mirada constructivista. Revista Sinéctica, No. 25, agosto 2004-enero 2005, sección separata, 1-24. Recuperado el 30 de 
septiembre de 2011 en: http://portal.iteso.mx/portal/page/portal/Sinectica/Historico/ Numeros_anteriores05/025/25\%20Cesar\%20Coll-Separata.pdf

CUESTA, M. Y HERRERO, F. J. (2010). Introducción al muestreo. Depto. de Psicología, Universidad de Oviedo. Recuperado el 15 octubre de 2011 en: http://www.psico.uniovi.es/Dpto_Psicologia/metodos/tutor.7

DUART, J. M., GIL, M., PUJOL, M. Y CASTAÑO, J. (2008). La universidad en la sociedad red. Usos de Internet en Educación Superior. Barcelona: Ariel.

GÓMEZ, A. Y MARTíNEZ, M. (2008). La educación en móvil(idad). Comunicar, 16 (31), 699-708. Recuperado el 22 de enero de 2012 en: http://www.revistacomunicar.com/index.php?contenido=detalles\&numero=31\&arti culo $=31-2008-93$

HERRERA, J.A., LOZANO, F.G. Y RAMÍREZ, M.S. (2008). Competencias aplicadas por los alumnos para el uso de dispositivos m-learning. Memorias del XVII Encuentro Internacional de Educación a Distancia. Virtualizar para educar. Guadalajara, Jalisco.

HUSSAIN, I. Y ADEEB, M. (2009). Role of mobile learning in promoting campuswide learning environment. The Turkish Online Journal of Educational Technology, 8 (3), 48-56.

KENNY, R., VAN NESTE-KENNY, J., PARK, C., BURTON, P. Y MEIERS, J. (2009). Mobile Learning in Nursing Practice Education: Applying Koole's FRAME Model. Journal of Distance Education Revue de L'Education a Distance, 23 (3), 75-96.

KOOLE, M. (2006).The Framework for the Rational Analysis of Mobile Education (FRAME) Model: An Evaluation of the Mobile Devices for Distance Education. Tesis para optar al grado de Maestro en Educación a Distancia. Athabasca University, Alberta, Canadá. Recuperado el 15 de marzo de 2010 en: http://auspace.athabascau.ca:8080/dspace/bitstream/2149/543/1/aaa_FINAL_VERS ION_mkoole_thesis_edited_May9_2006.pdf

KOOLE, M. (2009). A Model for Framing Mobile Learning. En: Ally, M. (Ed.). Mobile Learning: Transforming the Delivery of Education \& Training. Edmonton, AB: AU Press, Athabasca University.

KUKULSKA-HULME, A. (2009). Will mobile learning change language learning? $\operatorname{ReCALL}, 21(2), 157.165$.

KUKULSKA-HULME, A. Y TRAXLER, J. (2005). Mobile teaching and learning. En: Kukulska-Hulme, A. y Traxler, J. (Ed.) Mobile Learning. A Handbook for educators and trainers. Abingdon, Oxon: Routledge Taylor \& Francis Group.

LAURILLARD, D. (2007). Pedagogical forms for Mobile Learning. En: Pachler, N. (Ed.) Mobile learning: towards a research agenda. London: WLE Center, loE. Recuperado el 30 de abril de 2012 en: http://eprints.ioe.ac.uk/627/1/Mobile_C6_Laurillard.pdf 
LUNSFORD, J. (2010). Using Handheld Technologies for Student Support: A Model. Journal of Research Center for Educational Technology, 6 (1), 55-69.

NAISMITH, L., LONSDALE, P., VAVOULA, G. Y SHARPLES, M. (2005). Literature Review in Mobile Technologies and Learning. Report 11. FutureLab Series. University of Birmingham. Disponible en: http://www2.futurelab.org.uk/ resources/documents/lit_reviews/Mobile_Review.pdf

PETIT, J. Y KUKULSKA-HULME, A. (2006). Going to the grain: Mobile devices in practice. Australasian Journal of Educational Technology, 23 (1), 17-33. Consultado el 15-11-10 en: http://oro.open.ac.uk/7118/1/Pettit_and_KukulskaHulme_ajet-p17-33.pdf

O'DWYER, L., RUSSELL, M. Y BEBELL, D. (2005). Identifying teacher, school, and district characteristics associated with middle and high school teachers' use of technology: a multilevel perspective. Journal of educational computing research, 33 (4), 369-393.

RAMOS, A.I., HERRERA, J.A. Y RAMIREZ, M.S. (2010). Desarrollo de habilidades cognitivas con aprendizaje móvil: un estudio de casos. Comunicar, 17 (34), 201209.

RUSSELL, M., O'DWYER, L., BEBELL, D. Y MIRANDA, H. (2004). Technical report for the USEIT study. Boston, MA: Boston College, Technology and Assessment Study Collaborative. Recuperado el 06 de junio de 2007, en: http://escholarship.bc.edu/cgi/viewcontent.cgi?article=1024\&context=intasc

SHARPLES, M. (2005). Learning as Conversation: Transforming Education in the Mobile Age. En: Proceedings of Conference on Seeing, Understanding, Learning in the Mobile Age (pp. 147-152). Budapest, Hungría. Recuperado el 8 de mayo de 2010 en: http://www.fil.hu/mobil/2005/sharples_final.pdf

SHARPLES, M. (2007). How can we address the conflicts between personal informal learning \& traditional classroom education?. En: Sharples, M. (Ed.). Big Issues in Mobile Learning. Report of a workshop by the Kaleidoscope Network of Excellence Mobile Learning Initiative. Learning Science Research Institute. University of Nottingham.

TRAXLER, J. (2007). Defining, Discussing, and Evaluating Mobile Learning: The moving finger writes and having writ... International Review of Research in Open and Distance Learning, 8 (2), 1-12.

TRAXLER, J. (2009). Learning in a Mobile Age. International Journal of Mobile and Blended Learning, 1 (1), 1-12.

UNESCO (2012). Turning on Mobile Learning in Latin America. Illustrative Initiatives and Policy Implications. Working Papers Series on Mobile Learning. Consultado el 2011-2012 en: http://unesdoc.unesco.org/images/0021/002160/216080e.pdf

WALKER, K. (2007). Mapping the landscape of mobile learning. En: Sharples, M. (Ed.) (2007). Big Issues in Mobile Learning. Report of a workshop by the 
Kaleidoscope Network of Excellence Mobile Learning Initiative. Learning Science Research Institute. University of Nottingham.

\section{Correspondencia con los autores}

Patricio HENRÍQUEZ-RITCHIE

Instituto de Investigación y Desarrollo Educativo, Universidad Autónoma de Baja California

Km. 103 Carretera Tijuana-Ensenada

Ensenada, Baja California, México

Tel. (646) 1750707

henriquezritchie@hotmail.com

Coral GONZÁLEZ BARBERA

Dpto. Métodos de Investigación y Diagnóstico en Educación, Facultad de Educación, Universidad Complutense de Madrid

Calle Rector Royo Villanova s/n

Madrid, España

Tel. (34) 913946112

coralgnzlz3@gmail.com

Javier ORGANISTA-SANDOVAL

Instituto de Investigación y Desarrollo Educativo,

Universidad Autónoma de Baja California

Km. 103 Carretera Tijuana-Ensenada

Ensenada, Baja California, México

Tel. (646) 1750707

javor@uabc.edu.mx 